\title{
Evaluation of Diagnostic Criteria and Incidence of Acute Mountain Sickness in Preverbal Children
}

\author{
Michael Yaron, MD; Susan Niermeyer, MD; Kjell Norwood Lindgren; Benjamin Honigman, MD \\ From the University of Colorado Health Sciences Center, Department of Surgery, Division of Emergency Medicine, and the Colorado \\ Emergency Medicine Research Center, Denver, CO (Drs Yaron and Honigman); the University of Colorado Health Sciences Center, \\ Department of Pediatrics, Denver, CO (Dr Niermeyer); and the University of Colorado Health Sciences Center, School of Medicine, Denver, \\ CO (Mr Lindgren).
}

\begin{abstract}
Objective.-The Children's Lake Louise Score (CLLS) established the diagnostic criteria for acute mountain sickness (AMS) in preverbal children. Prospective application of the CLLS and interobserver agreement for the score had not been evaluated in a controlled trial. A study of children and their parents was used to evaluate the CLLS and determine the incidence of AMS in preverbal children.

Methods. - A prospective, controlled trial. Children $\geq 3$ months and $\leq 36$ months old and their parents living below $1645 \mathrm{~m}$ were studied over 7 separate days. The CLLS, measured daily by the mother as well as by the father on days 5 and 6 , is the sum of scores for fussiness (FS), eating (E), playfulness (P), and sleep (S). Children were studied on days 1 and 2 at home, on day 3 after travel without altitude gain to a hotel, on day 4 at home, on days 5 and 6 at a hotel at $3109 \mathrm{~m}$, and on day 7 at home. Using our previous criteria, AMS was diagnosed if the CLLS was $\geq 7$ with both the FS $\geq 4$ and the $\mathrm{E}+\mathrm{P}+\mathrm{S} \geq 3$. Agreement between mothers' and fathers' CLLS values was measured with the kappa statistic (к). Adults were also evaluated for AMS by the CLLS on days 5 and 6 .

Results.- Thirty-seven children (mean age $\pm \mathrm{SD}=16.5 \pm 10.5$ months; 21 girls) participated, and AMS occurred in 7 of them (19\%; 95\% CI, 8.35\%). Among 33 adults, 8 (24\%; 95\% CI, 9.39\%) had AMS. Although the agreement of the parents on the CLLS components was poor, the agreement on the classification of AMS between mother and father was excellent $(\kappa=.67 ; P<.001)$, with both parents' scores exceeding the CLLS threshold for AMS.

Conclusions. - In this prospective trial, parents demonstrated excellent interobserver agreement for independent use of the CLLS to detect AMS. Consistent with our previous retrospective study, the incidence of AMS at moderate altitude in preverbal children (19\%) was similar to that in adults (24\%).
\end{abstract}

Key words: acute mountain sickness, pediatric, infant, altitude

\section{Introduction}

Millions of individuals travel to regions of moderate altitude (2500-3200 m) throughout the world annually, and many of these are families traveling with very young children. The incidence of acute mountain sickness (AMS) in adults traveling to moderate altitudes has been

Abstract presented in part at the Society of Academic Emergency Medicine Annual Meeting, San Francisco, CA, from May 22 to 25, 2000, and the Wilderness Medical Society Annual Meeting, Park City, UT, from August 8 to 12, 2000.

Corresponding author: Michael Yaron, MD, University of Colorado Health Sciences Center, Division of Emergency Medicine, Box B-215, 4200 E 9th Ave, Denver, CO 80262 (e-mail: michael.yaron@uchsc. edu). found to be approximately $25 \% .^{1}$ This incidence may vary widely depending on rate of ascent, final altitude gained, and individual susceptibility. ${ }^{2-4}$

Children traveling with their families to moderate altitudes also experience AMS. ${ }^{5}$ Clinicians in the ski resorts of the western United States are often presented with fussy, irritable infants and parents concerned about the behavioral changes seen in their children shortly after arrival to moderate altitude. Making a diagnosis of AMS in these preverbal children is difficult for a number of reasons. One fundamental reason is the inability to elicit an accurate history, particularly for the presence of a headache, the cardinal symptom of AMS in the adult. Because a self-reported history is impossible in this age 
group, we recently developed the Children's Lake Louise Score (CLLS), on the basis of behavioral observations of the child by the parent, to establish the diagnostic criteria for AMS in preverbal children. ${ }^{6}$ The score incorporates fussiness as the equivalent of headache, eating disturbances as the equivalent of gastrointestinal symptoms, playfulness as an index of fatigue and/or dizziness, and ability to nap or sleep through the night as an indicator of sleep disturbance. This score was developed retrospectively, and no subsequent prospective application or analysis of reproducibility was performed. This score was also developed using a 4-hour stay at altitude and thus may have underestimated the incidence of AMS in the selected population.

The purpose of this investigation was to apply the CLLS in a prospective fashion, assess the reproducibility of this score by measuring interobserver agreement, and assess the incidence of AMS in both adults and preverbal children during altitude exposure, which included overnight stays.

\section{Methods}

In this prospective trial, subjects were recruited from the Denver, CO $(1610 \mathrm{~m})$, metropolitan area. Parents with twin children were enrolled to increase the number of subjects studied. Twin and triplet children, $\geq 3$ months and $\leq 36$ months of age, and their parents or guardians, with permanent residence at or below $1645 \mathrm{~m}$ were included. Children were excluded from the study if they had any acute medical illness or any significant underlying medical problem. Any child who developed symptoms of AMS or irritability was examined by one of the investigators for the presence of any other illness.

Prior to the beginning of the study, an investigator met with the parents to discuss the study protocol and to train the parents in the application of the CLLS. The CLLS is the sum of scores for fussiness (FS), eating (E), playfulness $(\mathrm{P})$, and sleep $(\mathrm{S})$, as shown in the Figure. Each child served as his or her own control. The study was designed to control for the effects of travel, a change in surroundings, and a change in altitude. Measurements for each child were made over 7 separate days. The CLLS was measured daily after breakfast by the mother and also by the father on days 5 and 6. Days 1 and 2 involved measurements at home. Day 3 involved travel without altitude gain to a hotel for an overnight stay. All subjects traveled by ground in their own vehicles from the Denver area. Ground travel time was approximately 20 to 30 minutes for all subjects. On day 4, the subjects returned home; on days 5 and 6 , the subjects ascended to a similar hotel at $3109 \mathrm{~m}$; and on day 7, the subjects returned home. Ground travel time to the second hotel
Table 1. Subject characteristics of children and their parents

\begin{tabular}{lccc}
\hline \multicolumn{1}{c}{ Subjects } & Female & Male & Total \\
\hline Childen $(n)$ & & & \\
Age (mo) & $19.2 \pm 11.3$ & $12.9 \pm 8.2$ & $16.5 \pm 10.5$ \\
Twins & 18 & 16 & 34 \\
Triplets & 3 & 0 & 3 \\
Premature birth & 15 & 12 & 27 \\
Adults $(n)$ & 17 & 16 & 33 \\
Age (y) & $35.7 \pm 6.1$ & $35.0 \pm 4.7$ & $35.4 \pm 5.4$ \\
& & & \\
\hline
\end{tabular}

was approximately 1 hour, 45 minutes for all subjects. Using our previous criteria, AMS was diagnosed if the CLLS was $\geq 7$ with both the FS $\geq 4$ and the $\mathrm{E}+\mathrm{P}+$ $S \geq 3$. The diagnosis of AMS for each child was based on the CLLS generated by the primary care provider for that child. In our cohort, the primary care provider was always the mother. The fathers were present with their children on days 5 and 6 . To assess adults for the presence of AMS, they completed the CLLS on days 5 and $6 .^{7}$ Interobserver agreement of independent scoring by the mother and father was measured from data on days 5 and 6 with the kappa statistic (к). All means are reported \pm SD.

Before participation in the protocol, the parents of all child subjects signed an informed consent. The Colorado Multiple Institutional Review Board approved this investigation.

\section{Results}

Thirty-seven children, including 1 set of triplets and 17 twin pairs, participated. Seventeen mothers and 16 fathers participated; all were the biological parents of their children. One family included both a set of twins and a set of triplets. Further demographic data for children and adults are included in Table 1. Twenty-seven of the 37 children were born at gestational ages between 32 and 37.5 weeks. None of these children required ventilator support at birth. None had been diagnosed with chronic lung disease or required supplemental oxygen $>1$ month of life; all were free of apparent intercurrent viral infections in the 2 weeks before ascent to altitude.

Data for comparison of agreement between mothers' and fathers' independent CLLS and component scoring were available for 35 children (Table 2). One mother of 2 children was unmarried. This analysis revealed the following $\kappa$ values: CLLS $=.13(P<.02), \mathrm{FS}=.20(P$ $<.005), \mathrm{S}=.40(P<.001), \mathrm{P}=.53(P<.002)$, and 


\title{
Children's Lake Louise Score (CLLS)
}

\begin{abstract}
Fussiness is defined as a state of irritability that is not easily explained by a cause, such as tiredness, hunger, teething or pain from an injury or ear infection. Fussy behavior may include crying, restlessness, or muscular tension. Please rate your child's typical fussy behavior during the last 24 hours without the benefit of your intervention.
\end{abstract}

AMOUNT OF UNEXPLAINED FUSSINESS

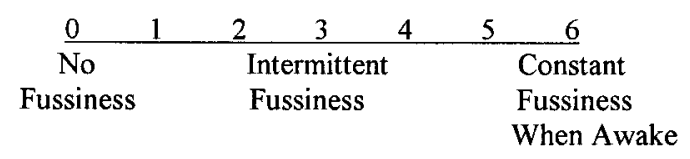

INTENSITY OF FUSSINESS

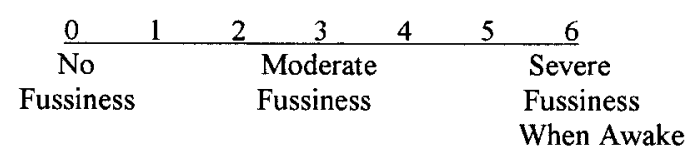

FUSSINESS SCORE (FS) $=$ Amount + Intensity

\begin{abstract}
0 -Normal
1 - Slightly less than normal

2-Much less than normal

3 -Vomiting or not eating
\end{abstract}

RATE HOW WELL YOUR CHILD HAS EATEN TODAY (E)

RATE HOW PLAYFUL YOUR CHILD IS TODAY (P)

0-Normal

1 - Playing slightly less

2-Playing much less than normal

3-Not playing

RATE ABILITY OF YOUR CHILD TO SLEEP TODAY (S)
0-Normal
1-Slightly less or more than normal
2-Much less or more than normal
3-Not able to sleep

$\mathbf{C L L S}=\mathbf{F S}+\mathbf{E}+\mathbf{P}+\mathbf{S}$

Figure. The Children's Lake Louise Score (CLLS). The diagnosis of acute mountain sickness (AMS) requires a CLLS total $\geq 7$, with both the fussiness $(F S) \geq 4$ and the eating plus playfulness plus sleep $(E+P+S) \geq 3$.

$\mathrm{E}=.61(P<.001)$. Although the agreement of the parents on the CLLS components ranged from poor to good, the agreement on the classification of AMS between mother and father was excellent $(\kappa=.67 ; P<$ .001). This occurred because score values between parents for the CLLS, although different (not in agreement), were both above the threshold value of 7 for the CLLS, $\geq 4$ for the FS, and $\geq 3$ for $\mathrm{E}+\mathrm{P}+\mathrm{S}$, resulting in scores that exceeded the CLLS threshold for AMS. On each day, only 1 set of parents reported CLLS scores for their child that disagreed regarding the presence of
AMS. In both cases, the mother's assessment met the criteria for AMS, while the father's did not.

Nineteen percent (7) of the children had AMS (95\% CI, 8.35\%), and among 33 adults, 24\% (8) had AMS (95\% CI, 9.39\%). Six of the 7 children who developed AMS were sibling pairs. The mean gestational age of children who developed AMS was $34.8 \pm 2.4$ weeks, while that of the group without AMS was $36.4 \pm 2.9$ weeks $(P=.20)$. No scores consistent with AMS were seen on any of the control days before ascent. All of the children with AMS after ascent had resolution of their 
Table 2. CLLS totals and AMS classification according to fathers and mothers

\begin{tabular}{|c|c|c|c|c|c|c|c|}
\hline \multicolumn{4}{|c|}{ Day 5} & \multicolumn{4}{|c|}{ Day 6} \\
\hline Father & Mother & Father & Mother & Father & Mother & Father & Mother \\
\hline$C L L S$ & $C L L S$ & $A M S$ & $A M S$ & $C L L S$ & $C L L S$ & $A M S$ & $A M S$ \\
\hline 2 & 7 & No & Yes & 11 & 13 & Yes & Yes \\
\hline 2 & 5 & No & No & 5 & 7 & No & Yes \\
\hline 2 & 3 & No & No & 0 & 4 & No & No \\
\hline 4 & 2 & No & No & 0 & 3 & No & No \\
\hline 8 & 4 & No & No & 14 & 15 & Yes & Yes \\
\hline 2 & 2 & No & No & 2 & 8 & No & No \\
\hline 1 & 2 & No & No & 2 & 2 & No & No \\
\hline 0 & 2 & No & No & 2 & 2 & No & No \\
\hline 3 & 8 & No & No & 3 & 2 & No & No \\
\hline 1 & 5 & No & No & 5 & 2 & No & No \\
\hline 0 & 2 & No & No & 4 & 2 & No & No \\
\hline 2 & 0 & No & No & 4 & 5 & No & No \\
\hline 6 & 5 & No & No & 7 & 4 & No & No \\
\hline 0 & 0 & No & No & 0 & 0 & No & No \\
\hline 0 & 0 & No & No & 0 & 0 & No & No \\
\hline 6 & 5 & No & No & 0 & 4 & No & No \\
\hline 5 & 6 & No & No & 0 & 5 & No & No \\
\hline 3 & 4 & No & No & 1 & 3 & No & No \\
\hline 4 & 4 & No & No & 4 & 3 & No & No \\
\hline 7 & 6 & No & No & 3 & 2 & No & No \\
\hline 2 & 8 & No & No & 2 & 5 & No & No \\
\hline 7 & 8 & No & No & 5 & 5 & No & No \\
\hline 3 & 6 & No & No & 4 & 5 & No & No \\
\hline 9 & 9 & Yes & Yes & 3 & 2 & No & No \\
\hline 13 & 12 & Yes & Yes & 9 & 2 & No & No \\
\hline 9 & 8 & No & No & 12 & 13 & Yes & Yes \\
\hline \multirow[t]{3}{*}{10} & 7 & No & No & 12 & 13 & Yes & Yes \\
\hline & 1 & & No & 3 & 2 & No & No \\
\hline & 3 & & No & 1 & 2 & No & No \\
\hline 5 & 4 & No & No & 3 & 3 & No & No \\
\hline 6 & 8 & No & No & 5 & 3 & No & No \\
\hline 4 & 4 & No & No & 2 & 2 & No & No \\
\hline 3 & 3 & No & No & 2 & 2 & No & No \\
\hline 7 & 1 & No & No & 9 & 4 & No & No \\
\hline 1 & 0 & No & No & 3 & 1 & No & No \\
\hline
\end{tabular}

*CLLS indicates Children's Lake Louise Score; AMS, acute mountain sickness. Children who met the criteria for AMS on either day are noted in bold type. One father was absent on day 5. Three children had AMS on Day 5, and 4 more had AMS on day 6.

symptoms and normalization of the CLLS shortly after their descent and return home.

\section{Discussion}

In this prospective trial, the CLLS was able to reliably diagnose AMS in preverbal children after acute, moderate-altitude exposure and to differentiate AMS from behavioral symptoms due to travel. The CLLS is reproducible by independent observers, as evidenced by excellent interrater agreement between parents scoring their children. Throughout the 7 days of measurement, no scores consistent with AMS were recorded on any nonascent days, providing further evidence that the CLLS appears to measure the presence of AMS. Nineteen percent of the preverbal children and $24 \%$ of the adults traveling from $1610 \mathrm{~m}$ to $3109 \mathrm{~m}$ developed AMS.

Determining the cause of an unhappy crying baby presents a challenge to both parents and clinicians. Changes in routine and environment can disturb a child and result in behavioral alterations. The changes in surroundings 
caused by travel alone may be responsible for alterations of appetite, playfulness, sleep, and fussy behavior in a child. Our original retrospective study controlled for the environmental change resulting from travel alone. ${ }^{6}$ Baseline measurements of the CLLS at home were obtained before any travel. These were compared with measurements made after travel with and without ascent. The threshold value for normal behavioral variation with and without travel was thus established and is supported by the current study, which included overnight stays away from home. If this threshold value is exceeded, the diagnosis of AMS may be made.

In very young children who develop fussiness after recent ascent to high altitude, a broad differential diagnosis must be entertained, as other underlying illnesses may mimic AMS. If, after recent ascent to altitude, one observes the development of fussiness with the concurrent presence of alterations of appetite, playfulness, and sleep, the placement of altitude illness high on the list of the differential diagnoses is warranted. While the CLLS may help identify the presence of AMS, it cannot be used to exclude the presence of other illnesses. Otitis and upper respiratory infections are the most likely conditions to be confused with AMS; however, less common but more serious conditions, such as intussusception or central nervous system infection, may also present with excessive irritability. Fever is an important sign that is inconsistent with AMS. In our study, the diagnosis of AMS was supported by the resolution of abnormal behavior and pathologic CLLS scoring after the descent and return home in all of our subjects with AMS.

Some limitations of the present study must be considered. Our subjects lived in the Denver metropolitan region at an altitude of $1610 \mathrm{~m}$. Had the subjects traveled from sea level to moderate altitude, the incidence of AMS would undoubtedly have been greater in both children and adults. Six of the subjects who developed AMS were siblings from 3 families. When analyzing data from pairs of siblings who share the same environment, one must consider the effect of one twin on the other. For example, if one sibling begins to cry and fuss, the other may become influenced by this behavior and experience similar distress. It is not clear if alterations of appetite, playfulness, and sleep in one child could influence his or her sibling. This influence could also be due to inherited traits shared by twins. The small number of subjects participating in this study makes analysis of the inheritability of traits impossible. ${ }^{8}$

Multifetal gestations are associated with a higher than normal incidence of preterm delivery. Five twin pairs were born at term; however, the rest of the children in the study were born slightly to moderately preterm (3237.5 weeks' gestation). No significant difference was noted in the mean gestational age of children who developed AMS compared to the group without AMS. While all of the 7 children diagnosed with AMS had a history of prematurity, none required ventilator support, received prolonged supplemental oxygen ( $>1$ month), or evidenced pulmonary hypertension or chronic lung disease. Premature infants who have required mechanical ventilation may show long-lasting alterations in pulmonary function, ${ }^{9}$ and perinatal diagnosis of pulmonary hypertension has been associated with hyperreactivity of the pulmonary vascular bed in response to high-altitude hypoxia into adult life. ${ }^{10}$ However, such alterations in function, as well as antecedent viral infections, are linked more closely with high-altitude pulmonary edema than with AMS. ${ }^{10,11}$

In our study population, parents did not have consistent agreement regarding the subscore components of the CLLS. This resulted in CLLSs that were in poor agreement. Because these scores are subjective assessments of a child's behavior, one would not expect precise agreement between independent observers. To make the diagnosis of AMS, however, a threshold value of CLLS components must be exceeded. These criteria for the presence or absence of AMS were met or exceeded with perfect agreement for all but 1 pair of parents on days 5 and 6 of the study.

\section{Conclusion}

In this prospective trial, after ascent to moderate altitude, the diagnosis of AMS in preverbal children can be made by the CLLS. Parents demonstrated an excellent degree of interobserver agreement for independent use of the CLLS. Consistent with our previous retrospective study, the incidence of AMS at moderate altitude in preverbal children $(19 \%)$ is similar to that in adults $(24 \%)$.

\section{Acknowledgments}

The authors wish to thank Lance Jobe, MD, Daniel Bissell, and Jeff Field for their technical assistance. This study was funded in part by the Wilderness Medical Society, Colorado American College of Emergency Physicians, and the Colorado Emergency Medicine Research Center.

\section{References}

1. Honigman B, Theis MK, Koziol-McLain J, et al. Acute mountain sickness in a general tourist population at moderate altitudes. Ann Intern Med. 1993;118:587-592.

2. Hackett PH, Rennie ID, Levine HD. The incidence, importance, and prophylaxis of acute mountain sickness. Lancet. 1976;2:1149-1154. 
3. Moore LG, Zamudio S, Curran-Everett L, et al. Genetic adaptations to high altitude. In: Wood SC, Roach RC, eds. Sport and Exercise Medicine. New York, NY: Marcel Decker; 1994:225-262.

4. Ross RT. The random nature of cerebral mountain sickness. Lancet. 1985;1:990-991.

5. Theis MK, Honigman B, Yip R, McBride D, Houston CS, Moore LG. Acute mountain sickness in children at 2835 meters. Am J Dis Child. 1993;147:143-145.

6. Yaron M, Waldman N, Niermeyer S, Nicholas R, Honigman B. The diagnosis of acute mountain sickness in preverbal children. Arch Pediatr Adolesc Med. 1998;152: 683-687.

7. Roach RC, Bärtsch P, Oelz O, Hackett PH. The Lake Lou- ise acute mountain sickness scoring system. In: Sutton JR, Houston CS, Coates G, eds. Hypoxia and Molecular Medicine. Burlington, VT: Queen City Press; 1993:272-274.

8. Martin N, Eaves L, Kearsey M, Davies P. The power of the classical twin study. Heredity. 1978;40:97-116.

9. Stahlman M, Hedvall G, Lindstron D, Snell J. Role of hyaline membrane disease in production of later childhood lung abnormalities. Pediatrics. 1982;69:572-576.

10. Sartori C, Allemann Y, Trueb L. Augmented vasoreactivity in adult life associated with perinatal vascular insult. Lancet. 1999;353:2205-2207.

11. Durmowicz AG, Noordeweir E, Nicholas R, Reeves JT. Inflammatory processes may predispose children to highaltitude pulmonary edema. J Pediatr. 1997;130:838-840. 